\title{
Kinematic Analysis of the Movement of Standing Up from and Sitting Down on a Chair
}

\section{Takatomo Mine*}

Department of Orthopaedic Surgery and Rheumatology, National Hospital Organization Kanmon Medical Center, Shimonoseki, Japan

\begin{abstract}
Standing up from and sitting down on a chair is one of most important movements for daily living. The decrease of these activities complicated with aging or brain disease is an important issue. TKA has a beneficial effect on knee function. TKA has been designed to restore the knee to a performance level close to that of a normal knee. During standing up from and sitting down on a chair, the motion patterns are different from normal knee motions. However, Post-TKA knee joint stability between the minimum and shallow knee flexion was good.
\end{abstract}

Keywords: Standing and sitting motion; Total knee arthroplasty; Posterior stabilized total knee arthroplasty; Post-cam mechanism

There are many types of movements used in daily activities. Gait and stair stepping and standing up from and sitting down on a chair are necessary for daily living. Particularly, standing up from and sitting down on a chair is one of most important movements for daily living. The decrease of this activity complicated with aging or brain disease is an important issue. With aging, standing up from and sitting down on a chair are challenging tasks for many elderly people. The differences between young and elderly subjects are related to deficits in postural control ability [1]. Mourey reported that there is a relationship between changes in the motor control of the task, which appeared during periods of potential postural instability and the effects of aging on postural instability [2]. Reisman reported that during standing up from and sitting down on a chair, a more variable vertical center of mass motion occurred. An enhanced use of goal-equivalent joint combinations under challenging task conditions, whether standing up or sitting down on a chair, is important [3]. For good performance of these movements, dorsiflexion of the ankle joint, a good position of the pelvis and good function of the knee are necessary. In the knee joint, many people contract osteoarthritis with aging. Osteoarthritis has a large effect on knee function due to pain, a decrease of muscle strength and impairment of the structure. Patients that have severe symptoms often undergo total knee arthroplasty (TKA). TKA can improve the knee function, but cannot recover it to normal function. Knee implants partially replace the function of the lost structure's intrinsic constraints, including the shapes of the articular surface, ligaments and guided motion of tibial bearings. Many knee implants have been designed and the main designs of knee prosthesis are mobile and fixed-bearing TKA. In fixed-bearing TKA,

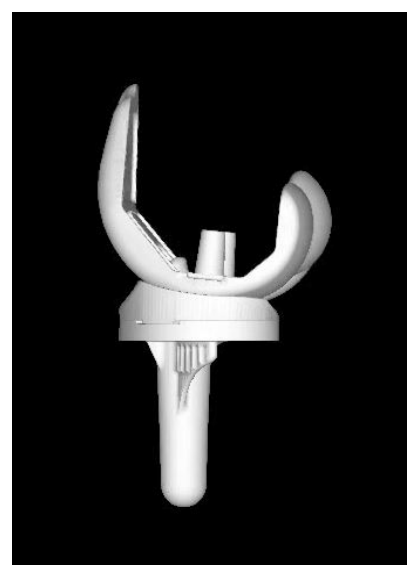

Figure 1: Computer-assisted design model in standing up. cruciate-retaining (CR) TKA and posterior-stabilized (PS) TKA have been designed to restore the knee to close to the function of a normal knee. Banks reported that many knees showed significantly different kinematics between the gait and stair activities, as well as differences from knees with other implant designs [4]. Thus, the implant design may influence the function of the knee after TKA.

The Bi-surface PS (Kyocera Medical Corporation, Japan) has a characteristic ball and socket joint structure in the mid-posterior portion of the femoral and tibial components. The post-cam mechanism is designed to start to function from $45^{\circ}$ to $60^{\circ}$. The ball \& Socket joint is designed to function as the main load-supporting surface from $90^{\circ}$ and to create anteroposterior stability [5]. We assessed in vivo knee motion patterns during standing up from and sitting down on a chair in patients with the Bi-Surface PS (Figures 1 and 2). During standing up from and sitting down on a chair, slight anterior femoral translation occurred during the transition

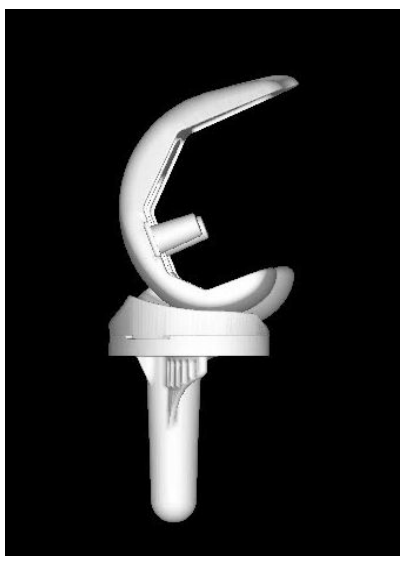

Figure 2: Computer-assisted design model in sitting down.

*Corresponding author: Takatomo Mine, M.D. PhD, Department of Orthopaedic Surgery and Rheumatology, National Hospital Organization Kanmon Medical Center, Shimonoseki, Japan, Tel: +81832411199; Fax: 81832411301; E-mail: minet@Kanmon-mc2.hosp.go.jp

Received October 03, 2017; Accepted October 13, 2017; Published October 20, 2017

Citation: Mine T (2017) Kinematic Analysis of the Movement of Standing Up from and Sitting Down on a Chair. Int J Neurorehabilitation 4: 290. doi: 10.4172/23760281.1000290

Copyright: () 2017 Mine T. This is an open-access article distributed under the terms of the Creative Commons Attribution License, which permits unrestricted use, distribution, and reproduction in any medium, provided the original author and source are credited. 
Citation: Mine T (2017) Kinematic Analysis of the Movement of Standing Up from and Sitting Down on a Chair. Int J Neurorehabilitation 4: 290. doi: 10.4172/2376-0281.1000290

from minimum to $30^{\circ}$ knee flexion; a medial pivot kinematic pattern was observed from $30^{\circ}$ to $60^{\circ}$ knee flexion and a bicondylar rollback kinematic pattern, in which both condyles moved backward in parallel, was seen from $60^{\circ}$ knee flexion to maximum knee flexion [6]. These motion patterns are different from normal knee motions [7,8] and are likely due to the post/ cam engagement. These kinematic patterns were observed more during the standing motion than during the sitting motion. Janssen reported that the ability to do the sit-to-stand movement, according to the reviewed literature, is strongly influenced by the height of the chair seat, use of armrests and foot position [9]. In our study, the height of the chair seat may be related to the results. However, the post-TKA knee joint stability between minimum and shallow knee flexion was good. TKA knee joint stability is also related to various factors such as muscular strength, ligament balance and component positioning. We think that these kinematic patterns will affect the long-term results. The relationship between these kinematic patterns and clinical results should be assessed in future research and longterm follow-up.

\section{References}

1. Mourey F, Pozzo T (1996) Effects of aging on standing up and sitting down. Kinematic analysis. C R Seances Soc Biol Fil 190: 603-611.
2. Mourey F, Pozzo T, Rouhier-Marcer I, Didier JP (1998) A kinematic comparison between elderly and young subjects standing up from and sitting down in a chair. Age Ageing 27: 137-146.

3. Reisman DS, Scholz JP, Schoner G (2002) Differential joint coordination in the tasks of standing up and sitting down. J Electromyogr Kinesiol 12: 493-505.

4. Banks SA, Hodge WA (2004) Design and activity dependence of kinematic in fixed and mobile-bearing knee arthroplasties. J Arthroplasty 19: 187-193.

5. Mine T, Hoshi K, Gamada K, Ihara K, Kawamura K, et al. (2016) In vivo kinematics of a unique posterior-stabilized knee implant during a stepping exercise. J Orthop Surg and Res 11: 18.

6. Mine T, Hoshi K, Gamada K, Ihara K, Kawamura K, et al. (2016) Kinematic analysis of posterior-stabilized total knee arthroplasty during standing up from and sitting down on a chair. J Orthop Surg Res 11: 142.

7. Freeman MA, Pinskerova V (2003) The movement of the knee studied by magnetic resonance imaging. Clin Orthop Relat Res 40: 35-43.

8. Johal P, Williams A, Wragg P, Hunt D, Gedroyc W (2005) Tibiofemora movement in the living knee. A study of weight bearing and non-weight bearing knee kinematics using 'interventional' MRI. J Biomech 38: 269-276.

9. Janssen WG, Bussmann HB, Stam HJ (2002) Determinants of the sit-to-stand movement: A review. Phys Ther 82: 866-879. 